\title{
IMPROVEMENT OF PRODUCTIVE AND REPRODUCTIVE PERFORMANCE OF DANDARAWI CHICKEN THROUGH FLASH LIGHT PROGRAM
}

\author{
M. F. A. Farghly
}

Department of Poultry Production, Faculty, of Agriculture, University of Assiut, (71516), Egypt

Email: farghly20002000@yahoo.com

\section{SUMMARY}

An experiment with Dandarawi chickens was designed to see the impact of flash light (FL) stimulation on productive and reproductive performance. Three hundred, four weeks old chicks were divided into 5 equal groups (3 replicates of 20 birds each) and housed in floor pens. The first group, the birds were subjected to 12 and 16 hrs common light (CL)/day during the growing and laying periods, respectively and were considered as the control group $(C)$. While, the second group (T1), birds were subjected to 12 hrs light (9 CL: 3 FL) and16 h light (12 CL:4 FL) during growing and laying periods, respectively. The third group (T2), birds were subjected to $12 \mathrm{hrs} \mathrm{light} \mathrm{(6} \mathrm{CL:6} \mathrm{FL)} \mathrm{and16} \mathrm{hrs} \mathrm{light}(8 \mathrm{CL}: 8 \mathrm{FL})$ during growing and laying periods, respectively. The fourth group (T3), birds were subjected to $12 \mathrm{~h}$ light (3 CL: $9 \mathrm{FL})$ and16 h light (4 CL:12 FL) during growing and laying periods, respectively. The fifth group (T4), birds were subjected to 12 and 16 hrs flash light (FL)/day during the growing and laying periods, respectively. Feed and water were available ad libtum and all the other conditions were the same during the experimental period. Results emanating from this study indicated that exposure of birds to flash light affected many of the studied traits and the effects were significant $(P \leq 0.05)$ on body weight, weight gain, feed conversion, liver, giblets, abdominal fat, total lipids, blood AST, H/L ratio, egg number, egg shell thickness, egg yolk index, age at sexual maturity, fertility, semen volume \& concentration, follicle number, testes and ovary percentages. While, there were no significant $(P>0.05)$ differences in most carcass traits, blood components (protein, cholesterol, ALT), shell strength, Haugh units, egg components, leg problems, plumage conditions, bone lengths, semen $\mathrm{pH}$, oviduct weight \& length and hatchability percentages. Finally, the economical efficiency of the birds reared on 12 hrs light (6 CL: 6 FL) during the growing period and birds provided with either $16 \mathrm{hrs}$ of light $(4 \mathrm{CL}: 12 \mathrm{FL}$ ) for table eggs, as well as (8 CL: $8 \mathrm{FL}$ ) or (4 CL:12 FL) for fertile eggs during laying periods were superior to that of birds in other groups. Light flashes program as bio-intermittent light might be economical alternative light to continuous or common light in sight and stimulation of internal organs or initiation of hormones release without negatively affecting the performance and welfare of chickens during growing and laying periods.

\section{Keywords: Flash light, productive and reproductive performance, Dandarawi chickens}

\section{INTRODUCTION}

It is necessary, searching for ways to enhance the productive efficiency of local chicken through some managerial tools and an adequate environment. Light is an important environmental and managerial factor that influences the growth, egg production, carcass traits, behavior and health, therefore, light manipulation is widely used to increase the productive and reproductive performances of chicken (Wineland, 2002, Prescott et al., 2003, Classen, et al., 2004; Lewis and Gous, 2006abc., Lewis et al., 2004; 2007, 2009ab, 2010 and Bayram and Özkan, 2010). Light is integral to sight and synchronize many essential functions, including stimulation of internal organs and initiation of hormone release and various metabolic steps that facilitate feeding and digestion (Olanrewaju et al., 2006). Poultry may receive light through pineal gland that has the ability to absorb light, penetrating the skull (Li and Howland 2003). Therefore, the pineal gland appears to translate environmental cues into melatonin secretions that are necessary for daily regulation of cardiopulmonary, reproductive, excretory, thermoregulatory, behavioral, and immune systems (Pang et al., 1996, Abbas et al., 2007 and Zawilska et al., 2007).
Chickens raised under intermittent light programs are likely to have higher serum melatonin levels than chickens raised under constant light programs. Exposure to light at night or during the dark period is accompanied by a significant decrease in melatonin levels (Navara and Nelson, 2007). Birds provided with long dark periods have fewer health related problems, including sudden death syndrome, spiking mortality and leg problems than those maintained in continuous or near continuous light (Apeldoorn et al., 1999 and Moore and Siopes, 2000). In the past, many producers have used constant or continuous light in chicken flocks in the belief that, birds can eat 24 hrs/day and grow faster. However, maximal benefit is obtained by rearing under a lighting regimen with a minimum dark period of 6 or $8 \mathrm{hrs}$ (Prescott et al., 2003), because it reduces metabolic problems, eye damage, physiological stress (Manser, 1996, Kliger et al., 2000 and Campo and Da'vila, 2002). During the dark period, heat production decreased by $25 \%$ in comparison with the light period and this decrease may result from depressed activity and resting or sleeping (Saiful et al., 2002). Therefore, most of the recent researches have focused on restricting or intermittent light regimens to improve productivity of chickens because the physical activity is very low 
during darkness and energy expenditure of activity is considerable (Rahimi et al., 2005). Use of biointermittent lighting program enhanced performance, lighting improved immune status, reduced room temperature and cost of electricity (Ohtani and Lesson 2000, Classen, 2004, Rahimi et al., 2005 and Olanrewaju et al., 2006).

To decide which chicken lighting program should be implemented, there are several critical factors a producer should consider in addition to the performance such as an economic (reduce electricity costs) and welfare standpoints. Lighting schedules can be characterized in a number of ways, including the number of hours or minutes of light and how many periods of darkness or pulses are included in each $24 \mathrm{~h}$ cycle. Light flashes can be widely used to improve production efficiency and as a way to reduce electricity consumption. The awareness of these major drawbacks and the associated financial losses led to a renewed interest in optimizing techniques (low cost) in chicken rearing management as providing birds a photoperiod involves light flashes. It is not known whether light flashes stimulation as bio-intermittent light makes any difference compared to continuous light stimulation which may be beneficial. Therefore, the objective of this experiment was to study the impact of using light flashes, as an economical alternative light source than common and continuous light for raising Dandarawi chickens.

\section{MATERIALS AND METHODS}

The present work was carried out at the Research Poultry Farm, Poultry Production Department,
Faculty of Agriculture, Assiut University. Three hundred (150 male and 150 female), four weeks old chicks were divided into 5 equal groups ( 3 replicates of 20 birds each) and housed in floor pens. The first group, the birds were subjected to 12 and $16 \mathrm{hrs}$ common light (CL)/day during the growing and laying periods, respectively and were considered as a control (C). While, the second group (T1), birds were subjected to $12 \mathrm{hrs}$ light (9CL: 3FL) and $16 \mathrm{~h}$ light (12CL: 4FL) during growing and laying periods, respectively. The third group (T2), birds were subjected to $12 \mathrm{hrs}$ light (6CL: 6FL) and $16 \mathrm{hrs}$ light (8CL: 8FL) during growing and laying periods, respectively. The fourth group (T3), birds were subjected to $12 \mathrm{hrs}$ light (3CL: 9FL) and16 hrs light (4CL: 12FL) during growing and laying periods, respectively. The fifth group (T4), birds were subjected to 12 and $16 \mathrm{hrs}$ light flash (FL)/day during the growing and laying periods, respectively. All sources of natural light were covered with heavy cotton black curtains and blackout plastic curtains which completely prevent any source of natural light. Light intensity measured at the middle of the room ranged between 25-30 lux. using incandescent bulbs. Flashing light regimens each one hour was 20 flashes/ minute. Feed and water were available ad libtum and all the other conditions were the same during the experimental period (4-36 weeks of age). Birds received growing and laying diets from 4-20 and 21-36 weeks of age, respectively. The composition and calculated analysis of the experimental diets are shown in Table (1).

Table 1. Composition and calculated analysis of experimental diet

\begin{tabular}{|c|c|c|}
\hline Ingredients & Growing (\%) & Laying (\%) \\
\hline Yellow corn & 64.0 & 69.5 \\
\hline Soybean meal $(44 \%)$ & 25.5 & 15.0 \\
\hline Concentrate & $8.0 *$ & $8.0 * *$ \\
\hline Salt & 0.20 & 0.10 \\
\hline Minerals & 0.20 & --- \\
\hline Premix & 0.30 & -- \\
\hline Bone meal & ---- & 0.4 \\
\hline Limestone & ---- & 7.0 \\
\hline Total & 100 & 100 \\
\hline \multicolumn{3}{|c|}{ Calculated analysis $* * *$} \\
\hline Protein (\%) & 21.0 & 17.4 \\
\hline ME ( KCal/ Kg diet) & 2893 & 2867 \\
\hline Calcium (\%) & 1.20 & 3.10 \\
\hline Available phosphorus (\%) & 0.55 & 0.37 \\
\hline
\end{tabular}

* Broiler concentrate $\quad * *$ Layer concentrate $\quad * * *$ Calculated according to NRC (1994).

Body weight (BW) and feed consumption (FC) were recorded weekly and calculated periodically every 2 weeks. Feed conversion ratio, (FCRg): (g feed/ g gain) was calculated biweekly by dividing the total feed consumed every 2 weeks $(\mathrm{g} / \mathrm{d} / \mathrm{h})$ in a pen by the total weight gain $(\mathrm{g} / \mathrm{d} / \mathrm{h})$ of its birds. Also, the feed conversion ratio values ( $\mathrm{g}$ feed/g egg mass, FCRe) were calculated periodically every four weeks, from 24 to 36 weeks of age. At 20 weeks of age, 6 birds per group ( 3 male and 3 female) were randomly chosen and slaughtered. The internal organs were removed while the heart, liver, empty gizzard, testes and ovary including the yellow follicles, were weighed. Each of head at the occipital bone, feet and shanks at the hock joints, wings at shoulder joints, neck close to the shoulder, breast, femurs and drumsticks were weighed as separate carcass parts. Each of carcass weight, drumsticks, femurs, breast, wings and back were calculated as percentages of pre-slaughter live body weight, while each of heart, 
liver, gizzard, giblets, testes and ovary were calculated as percentages of carcass weight.

Blood samples were collected at 6 weeks of age in heparinized tubes. The blood samples were centrifuged at $3000 \mathrm{rpm}$ for $15 \mathrm{~min}$ and plasma obtained were stored at $-20^{\circ} \mathrm{C}$ until analysis. Plasma total protein, albumin, total cholesterol and transaminase enzymes activities (AST and ALT) were determined colorimetrically using available commercial kits made by Spectrum Company. The globulin values were obtained by subtracting the values of albumin from the corresponding values of total protein. Differential leucocyte counts were determined using the conventional methods. Plumage scores were measured at 24 and 36 weeks of age. Three areas of the body were measured (head, neck and back) using a scale from 1(completely feathered) to 5 (featherless).

Egg weight, egg number and hen-day egg production (HDP) were counted and recorded periodically every four weeks, from 24 to 36 weeks of age. Cracked or broken eggs, and those laid on the litter floor were recorded separately. Age at sexual maturity (ASM) was calculated as the number of days at $50 \%$ egg production. Dead birds were recorded daily and expressed as percentage during the experimental period. During the period from 24 to 36 weeks of the experiment, 45 fresh-laid eggs were taken, every four weeks, from each group to measure egg quality characteristics. Egg weight was recorded to the nearest 0.1 gram on the same day of collection using electronic scale. The length and width of each egg were determined using a sliding caliper and their egg shape index $=$ (width of egg/length of egg) 100 was calculated. Shell strength was obtained by measuring the resistance of the egg shell to a gradually increasing horizontal force using egg cracking machine to the nearest $0.1 \mathrm{~kg}(\mathrm{~kg} / \mathrm{cm} 2)$ (Germany-Wazau). Shell thickness of the dried shell (without membranes) was measured using shell thickness apparatus (millimeters). The height of thick albumen and yolk were measured using a micrometer. Haugh unit values were calculated for each egg using the formula: Haugh unit $=100 \log (\mathrm{H}-$ $1.7 \times \mathrm{W} 0.37+7.6$ ) Where: $\mathrm{H}=$ the observed height of the albumen in millimeters and $\mathrm{W}=$ weight of egg (g). The diameter of yolk was measured, using a sliding caliper. The yolk was separated from the albumen and weighed. The yolk index was calculated by dividing (yolk's height/yolk's diameter) x100. Also, shells with membranes were dried and weighed to the nearest $0.01 \mathrm{gm}$. Three hatches were obtained from flock with 1:10 sex ratio at 28,32 and 36 weeks of age using Peterzime setter. Fertility and hatchability percentages were calculated as follow: Fertility $(\%)=($ Fertile eggs $) \times 100 /$ Total eggs set \& True hatchability $(\%)=($ Viable hatched chicks $) \times 100$ / fertile eggs.

Starting at $16 \mathrm{wk}$, each male was individually checked at intervals of 1 or $2 \mathrm{wk}$ for onset of semen production, by manual massage. Onset of mature semen production was evaluated by eye, and scored on a scale of 6 to 1 , as follows: where $6=$ shrunken cloacal exit, $5=$ extrusion of rudimentary penis, without semen production, $4=$ production of seminal fluid, 3 = production of yellow semen, $2=$ some indication of white semen, 1 = production of white semen. A score of 1 was given to represent achievement of fully mature semen production on the part of the male. At this time, age and body weight were obtained for the bird. Semen was collected into graduated collection tube to record the volume per ejaculate with an accuracy of $0.05 \mathrm{ml}$. After collection, tube was maintained at $38-40^{\circ} \mathrm{C}$ in a thermo flask. Semen $\mathrm{pH}$ was measured by comparative $\mathrm{pH}$ papers immediately after collection. Semen color: Semen color was scored from 1 to 3 (Where 1= milky, $2=$ creamy and $3=$ watery). Sperm concentration (millions per milliliter) was determined by the hemocytometer (Thoma) method (Salisbury et al., 1985).

Economical efficiency (EE): Feed cost per bird was calculated by multiplying mean FC per bird by the cost of $1 \mathrm{~kg}$ of diet. Bird price was calculated by multiplying mean bird weight by price of $1 \mathrm{~kg}$ of live weight. The net revenue per bird was estimated as the difference between the total income/bird (LE), (growth, table and fertile egg production) and the total costs of feed and light costs. Economical efficiency (EE) was estimated by dividing net revenue by total feed and light costs.

Statistical analysis: Data collected were subjected to ANOVA by applying the General Linear Models Procedure of SAS software (SAS institute, version $6.12,1996)$. Duncan (1955) was used to detect differences among means of different groups. Significance was set at the $5 \%$ level. The following model was used for analysis of variance:

$$
\mathbf{Y}_{\mathrm{ij}}=\boldsymbol{\mu}+\mathbf{S}_{\mathrm{i}}+\mathbf{e}_{\mathrm{ij}}
$$

Where: $Y_{i j}=$ observation, $\mu=$ overall mean, $\mathrm{Si}=$ treatment effect, $\mathrm{e}_{\mathrm{ij}}=$ experimental errors.

\section{RESULTS AND DISCUSION}

\section{Body weight (BW) and body weight gain (BWG):}

Results in Table (2) show that there was a significant effect $(\mathrm{P} \leq 0.05)$ of light flashes program on body weight at 14, 16, 18 and 20 weeks of age. At 16,18 and 20 weeks of age, birds under 12CL/day (C), 3FL+9CL/day (T1) or 6FL+6CL/day (T2) had similar body weights and were heavier than those exposed to $9 \mathrm{FL}+3 \mathrm{CL} /$ day (T3) or $12 \mathrm{FL} /$ day (T4). Light flashes regime showed a significant effect $(\mathrm{P} \leq 0.05)$ on daily weight gain at $12-14,14-16,16$ - 18 and 18 - 20 wks of age, indicating that birds exposed to 12CL/day, 3 FL+9CL/day or $6 \mathrm{FL}+6 \mathrm{CL} /$ day exhibited higher growth rate than those maintained under $9 \mathrm{FL}+3 \mathrm{CL} /$ day or $12 \mathrm{FL} /$ day. From the obtained results, it could be noticed that, control group (CL) and alternatives flashed light schedules (T1 and T2) had similar increasing in body weight and body weight gain. This increase in growth 
rate for birds reared under fewer hours of light flashes (3 or $6 \mathrm{hrs}$ of light flashes/12 hrs of light) compared to more hours of light flashes ( 9 or $12 \mathrm{hrs}$ of light flashes/12 hrs of light) may be due to less physiological stress and activity pattern which affects energy expenditure (Saiful et al., 2002). Light pulses plays a pivotal role as common light in stimulation of internal organs and initiation of hormone release (Houser and Huber-Eicher, 2004).

Melatonin is a hormone released from the pineal gland, during the dark hours of the day and stimulates lymphocytes proliferation as well as antibody production (Aperdoorn et al., 1999, Kliger et al., 2000, Abbas et al., 2007\& 2008). Long dark periods (12 hrs) could be a stress and a main factor inducing elevation in corticosterone level increasing proinflammatory cytokines, which inhibit growth (Johnson, 1997). In the intermittent light, the broilers eat to satiation in the light period and then do not expand much energy during dark period, causing greater body weight gain (Ingram and Hatten, 2000). So, the use of flash light may be a correction tool to bio-intermittent light. Lanson (1961) noted no significant effects on body weight between birds receiving different light regimens included continuous or flashing light.

The present results are partially similar to those reported by Al-Homidan and Petchey (2000), Rahimi et al. (2005), Bölükbasi and Emsen (2006), El-Fiky et al. (2008) and Amakiri et al. (2011) who reported that cumulative growth of the intermittent light group was similar to the continuous light group, revealing compensatory growth. The recovery of growth of broilers exposed to intermittent light could be attributed to low activity during dark period, better digestion of feed and less maintenance nutrient requirements (Rahimi et al., 2005). Also, Classen (2004); Rahimi et al, (2005); Bölükbasi and Emsen (2006). Lewis and Gous (2006 a\&b); Downs et al. (2006), Gharib, et al. (2008), Abbas et al. (2008) and Mahmud et al. (2011) indicated that intermittent light significantly affect body weight. Classen et al. (2004), Lien et al. (2009); Lewis et al. (2009a\&b); Lewis et al. (2010) and Bayraktar et al. (2012) indicated that early growth rate was significantly reduced by longer periods of darkness, but gain as well as final body weight was not affected by lighting programs. In contrast, Boon et al. (2000) found that longer photoperiods were associated with larger weight gains. Kuhn et al. (1996) reported that male broiler chickens reared in near continuous lighting and intermittent lighting repeatedly had higher growth rates than birds under a continuous light. Broilers reared under continuous light gained more weight than those exposed to intermittent or restricted light (Shutze, et al., 1996, Ingram and Hatten, 2000 and Tuleun et al., 2010).

\section{Feed consumption (FC) and feed conversion (FCR):}

No significant differences were found in the overall mean of FC among all groups (Table 3). However, at 12-14 weeks of age, the birds of T1 and T4 groups consumed significantly $(\mathrm{P} \leq 0.05)$ more feed than the control, T2 and T3 groups. It was found that the overall mean of feed conversion for growth (FCRg) was significantly $(\mathrm{P} \leq 0.05)$ better due to illumination of birds with $12 \mathrm{CL}$ and all FL programs except that of $12 \mathrm{FL} /$ day program. However, the overall mean of feed conversion for egg (FCRe) was significantly $(\mathrm{P} \leq 0.05)$ better under FL programs of 6 CL+ $6 \mathrm{FL} /$ day or 3CL+9FL/day. Light and dark cycles allow the bird to establish rhythmicity and synchronize many essential metabolic functions by melatonin hormone that influence heat production, feed/water intake patterns and digestion secretion (Aperdoorn et al., 1999 and Olanrewaju et al., 2006). It is assumed that the reduction of activity during darkness may result in lower heat production, higher feed efficiency or both. Chickens do not feed or drink during a long period of darkness (Rahimi et al., 2005) although they may feed during short 4-hour dark periods. Duve et al. (2011) indicated that broilers modify their feeding behaviour according to the prevailing light or dark schedule, so birds eat about $80 \%$ of their total feed intake during the light period and eat little during the dark period and then influence on feed intake, digestibility or metabolizability. Lanson (1961) noted no significant effects in feed consumption were noted between birds receiving light regimens included continuous flash light.

Our findings are in partial agreement with many previous reporters (Ohtani and Leeson, 2000; Oyedeji and Atteh 2005; Rahimi et al., 2005, Onbasilar et al., 2007; Lewis et al., $2009 \mathrm{a \& b}$ and Mahmud et al., 2011). They claimed that bio-intermittent light schemes significantly affect the feed consumption and feed conversion of chickens. Significant improvement in feed conversion have been recorded in broilers reared under intermittent light schedule compared to birds receiving long photoperiod (Classen, 2004; Rahimi et al., 2005; Bölükbasi and Emsen, 2006; Gharib, et al., 2008 and Lien et al., 2009). In contrast, some investigations have demonstrated that chickens exposed to continuous light increased feed consumption than those given period of light and darkness (Shutze et al., 1996). Also, Tuleun et al. (2010); Duve et al. (2011) and Amakiri et al. (2011) reported that feed conversion was not significantly different between continuous and limited lighting. Al-Homidan and Petchey (2000), Saiful et al. (2002); Gous and Cherry (2004); Oyedeji and Atteh (2005); Downs et al. (2006); Abbas et al. (2008) and El-Fiky et al. (2008) found that chicks reared under intermittent light showed no significant differences in feed consumption and feed conversion compared to those under continuous light. Also, Lewis et al. (2010) reported no significant differences in feed conversion for egg production. 


\section{Carcass quality:}

Results in Table (4), showed no significant differences for the percentages of dressed carcass, drumsticks, femurs, breast, heart and gizzard among all groups. However, the differences were significant $(\mathrm{P} \leq 0.05)$ for liver, giblets and abdominal fat percentages. It was found that the liver and giblets percentages were significantly lower due to $3 \mathrm{CL}+9$ FL or $12 \mathrm{FL} /$ day illumination compared to birds with $12 \mathrm{CL}, 3 \mathrm{FL}+9 \mathrm{CL}$ and 6FL+6CL/day. Also, abdominal fat percentages were significantly lower under $3 \mathrm{CL}+9 \mathrm{FL}$ and $12 \mathrm{FL} /$ day compared to 12 $\mathrm{CL} /$ day. Reducing photoperiod to 6 hours per day by flash light could be used as a tool for reducing abdominal fat, reducing sudden death syndrome and upgrading carcass quality of broilers. This result reflected the decrease in energy expenditure and change in metabolic process towards leanness due to low activity in the dark period and better nutrient utilization. The obtained results are in partial agreement with the findings of Buyse et al. (1996) and Rahimi et al. (2005) who, reported that intermittent lighting program reduced fat deposition, due to decrease abdominal fat percentage. Also, Oyedeji and Atteh (2005) found that there was significant reduction in abdominal fat of broilers exposed to only 6 hours of light per day compared to the usual 12 hours of light/day. However, El-Fiky et al. (2008) reported no significant difference was found among light regimes on abdominal fat. Downs et al. (2006) indicated that minimal effects of lighting were observed for carcass or part yields. However, they reported a subtle substitution effect for breast yield as influenced by lighting program. Al-Homidan and Petchey (2000), El-Fiky et al. (2008) and Lien et al. (2009) found that chicks reared under intermittent light showed significant improvements in carcass characteristics. El-Fiky et al. (2008) found that heart, liver and gizzard percentage were not affected by light regime. Similar results were obtained by Onbasilar et al. (2007) and Shariatmadari and Moghadamian (2007). On the other hand, Oyedeji and Atteh (2005) found a significant reduction in abdominal fat of broilers exposed to only 6 hours of light per day compared to the usual 12 hours of light per day. Also, Chen et al. (2007) found that photoperiod had no effect on absolute abdominal fat pad or breast muscle weight. Lewis et al. (2009b) reported that breast meat yield was unaffected by photoperiod in Cobb birds. Robinson et al. (1999) indicated that there were no differences in carcass composition between different light treatment groups.

Table 2. Means \pm SE of body weight and body weight gain as affected by flash light program

\begin{tabular}{|c|c|c|c|c|c|c|}
\hline \multirow{2}{*}{ Traits } & \multirow{2}{*}{$\begin{array}{c}\text { Age } \\
\text { (wks) }\end{array}$} & \multicolumn{5}{|c|}{ Treatments } \\
\hline & & $\mathrm{C}$ & T1 & $\mathbf{T 2}$ & T3 & T4 \\
\hline \multirow{10}{*}{$\begin{array}{c}\text { Body weight } \\
\text { (g) }\end{array}$} & 4 & $167.32 \pm 1.5$ & $169.00 \pm 1.8$ & $169.41 \pm 1.7$ & $167.98 \pm 1.7$ & $169.32 \pm 1.6$ \\
\hline & 6 & $238.42 \pm 3.3$ & $236.10 \pm 3.0$ & $234.02 \pm 3.8$ & $238.18 \pm 3.4$ & $236.55 \pm 3.6$ \\
\hline & 8 & $366.62 \pm 3.4$ & $370.33 \pm 2.7$ & $369.95 \pm 2.6$ & $364.85 \pm 5.2$ & $369.45 \pm 4.6$ \\
\hline & 10 & $552.23 \pm 6.1$ & $559.42 \pm 5.9$ & $558.32 \pm 5.8$ & $550.28 \pm 4.7$ & $549.38 \pm 5.6$ \\
\hline & 12 & $873.23 \pm 4.5^{\mathrm{b}}$ & $892.67 \pm 4.3^{\mathrm{a}}$ & $894.52 \pm 4.9^{\mathrm{a}}$ & $880.02 \pm 4.4^{\mathrm{ab}}$ & $870.88 \pm 6.9^{b}$ \\
\hline & 14 & $1131.55 \pm 9.3^{\mathrm{b}}$ & $1173.7 \pm 11.3^{\mathrm{a}}$ & $1172.77 \pm 9.1^{\mathrm{a}}$ & $1083.87 \pm 11.7^{\mathrm{c}}$ & $1131.52 \pm 9.9^{b}$ \\
\hline & 16 & $1312.33 \pm 9.3^{\mathrm{a}}$ & $1311.0 \pm 13.8^{\mathrm{a}}$ & $1310.37 \pm 10.5^{\mathrm{a}}$ & $1229.0 \pm 13.2^{\mathrm{b}}$ & $1205.83 \pm 12.3^{\mathrm{b}}$ \\
\hline & 18 & $1451.02 \pm 9.5^{\mathrm{a}}$ & $1459.23 \pm 8.1^{\mathrm{a}}$ & $1460.52 \pm 9.3^{\mathrm{a}}$ & $1366.8 \pm 14.9^{b}$ & $1317.08 \pm 9.1^{\mathrm{c}}$ \\
\hline & 20 & $1534.18 \pm 11.3^{\mathrm{a}}$ & $1556.4 \pm 10.2^{\mathrm{a}}$ & $1548.17 \pm 8.5^{\mathrm{a}}$ & $1462.3 \pm 16.8^{\mathrm{b}}$ & $1454.00 \pm 11.5^{\mathrm{b}}$ \\
\hline & 36 & $1638.32 \pm 12.4$ & $1668.51 \pm 9.8$ & $1672.32 \pm 10.6$ & $1625.44 \pm 11.1$ & $1610.44 \pm 12.4$ \\
\hline \multirow{8}{*}{$\begin{array}{c}\text { Body weight } \\
\text { gain } \\
\text { (g/bird/day) }\end{array}$} & $4-6$ & $5.08 \pm 0.23$ & $4.79 \pm 0.19$ & $4.62 \pm 0.24$ & $5.01 \pm 0.22$ & $4.80 \pm 0.21$ \\
\hline & $6-8$ & $9.16 \pm 0.30$ & $9.59 \pm 0.26$ & $9.71 \pm 0.29$ & $9.05 \pm 0.40$ & $9.49 \pm 0.26$ \\
\hline & $8-10$ & $13.26 \pm 0.51$ & $13.51 \pm 0.42$ & $13.45 \pm 0.46$ & $13.25 \pm 0.49$ & $12.85 \pm 0.40$ \\
\hline & $10-12$ & $22.93 \pm 0.50$ & $23.80 \pm 0.44$ & $24.01 \pm 0.47$ & $23.55 \pm 0.40$ & $22.96 \pm 0.44$ \\
\hline & $12-14$ & $18.45 \pm 0.63^{\mathrm{a}}$ & $20.07 \pm 0.72^{\mathrm{a}}$ & $19.87 \pm 0.58^{\mathrm{a}}$ & $14.56 \pm 0.70^{\mathrm{b}}$ & $18.62 \pm 0.6^{\mathrm{a}}$ \\
\hline & $14-16$ & $12.91 \pm 0.35^{\mathrm{a}}$ & $9.81 \pm 0.66^{b}$ & $9.83 \pm 0.31^{\mathrm{b}}$ & $10.37 \pm 0.38^{\mathrm{ab}}$ & $5.31 \pm 0.64^{c}$ \\
\hline & $16-18$ & $9.91 \pm 0.61^{\mathrm{ab}}$ & $10.59 \pm 1.00^{\mathrm{a}}$ & $10.73 \pm 0.78^{\mathrm{a}}$ & $9.84 \pm 0.85^{\mathrm{ab}}$ & $7.95 \pm 0.90^{\mathrm{b}}$ \\
\hline & $18-20$ & $5.94 \pm 0.64^{\mathrm{b}}$ & $6.94 \pm 0.47^{b}$ & $6.26 \pm 0.42^{\mathrm{b}}$ & $6.83 \pm 0.54^{\mathrm{b}}$ & $9.78 \pm 0.35^{\mathrm{a}}$ \\
\hline Overall mean & & $12.20 \pm 0.10^{\mathrm{a}}$ & $12.39 \pm 0.09^{\mathrm{a}}$ & $12.31 \pm 0.08^{\mathrm{a}}$ & $11.56 \pm 0.14^{b}$ & $11.47 \pm 0.10^{b}$ \\
\hline
\end{tabular}


Table 3. Means \pm SE of feed consumption (FC) and feed conversion (FCR) as affected by flash light program

\begin{tabular}{|c|c|c|c|c|c|c|}
\hline \multirow{2}{*}{ Traits } & \multirow{2}{*}{$\begin{array}{c}\text { Age } \\
(\text { wks })\end{array}$} & \multicolumn{5}{|c|}{ Treatments } \\
\hline & & $\mathbf{C}$ & T1 & T2 & T3 & T4 \\
\hline \multirow{9}{*}{$\begin{array}{c}\text { FC } \\
\text { (g/bird/day) } \\
\text { during } \\
\text { growing } \\
\text { period }\end{array}$} & $4-6$ & $25.63 \pm 0.61$ & $25.27 \pm 0.78$ & $25.47 \pm 0.68$ & $25.37 \pm 0.59$ & $25.43 \pm 0.70$ \\
\hline & $6-8$ & $29.50 \pm 1.78$ & $29.50 \pm 1.78$ & $28.37 \pm 1.24$ & $28.80 \pm 0.60$ & $30.13 \pm 1.16$ \\
\hline & $8-10$ & $34.70 \pm 0.92$ & $35.03 \pm 1.05$ & $34.51 \pm 1.45$ & $34.43 \pm 1.34$ & $35.77 \pm 1.23$ \\
\hline & $10-12$ & $42.80 \pm 1.47$ & $43.97 \pm 0.87$ & $42.00 \pm 1.31$ & $41.37 \pm 1.30$ & $44.00 \pm 1.14$ \\
\hline & $12-14$ & $46.70 \pm 0.57^{b}$ & $50.33 \pm 0.35^{\mathrm{a}}$ & $47.53 \pm 0.75^{b}$ & $46.50 \pm 0.67^{\mathrm{b}}$ & $50.73 \pm 0.84^{\mathrm{a}}$ \\
\hline & $14-16$ & $68.07 \pm 0.90$ & $68.87 \pm 0.73$ & $67.37 \pm 1.34$ & $68.17 \pm 0.55$ & $68.80 \pm 1.18$ \\
\hline & $16-18$ & $78.73 \pm 0.45$ & $78.87 \pm 0.73$ & $78.53 \pm 1.07$ & $78.10 \pm 0.64$ & $80.10 \pm 1.19$ \\
\hline & $18-20$ & $90.03 \pm 0.83$ & $91.13 \pm 0.73$ & $89.87 \pm 1.16$ & $89.13 \pm 1.27$ & $92.10 \pm 1.17$ \\
\hline & Overall mean & $52.02 \pm 0.27$ & $52.87 \pm 0.63$ & $51.71 \pm 0.29$ & $51.48 \pm 0.76$ & $53.38 \pm 0.79$ \\
\hline \multirow{5}{*}{$\begin{array}{c}\text { FC } \\
\text { (g/bird/day) } \\
\text { during laying } \\
\text { period }\end{array}$} & $20-24$ & $97.68 \pm 0.8$ & $96.42 \pm 2.0$ & $97.00 \pm 1.6$ & $95.73 \pm 0.6$ & $95.16 \pm 1.4$ \\
\hline & $24-28$ & $102.11 \pm 1.1$ & $101.40 \pm 1.5$ & $99.82 \pm 1.0$ & $99.86 \pm 1.3$ & $99.63 \pm 0.8$ \\
\hline & $28-32$ & $105.0 \pm 1.7$ & $104.2 \pm 1.2$ & $104.6 \pm 1.8$ & $103.8 \pm 1.6$ & $104.3 \pm 1.3$ \\
\hline & $32-36$ & $108.4 \pm 0.9$ & $108.0 \pm 2.1$ & $107.4 \pm 1.6$ & $107.4 \pm 1.2$ & $108.3 \pm 1.6$ \\
\hline & Overall mean & $103.30 \pm 0.40$ & $102.51 \pm 0.90$ & $102.21 \pm 0.30$ & $101.70 \pm 0.42$ & $101.85 \pm 0.61$ \\
\hline \multirow{9}{*}{$\begin{array}{c}\text { FCRg } \\
\text { (g feed/g gain) }\end{array}$} & $4-6$ & $5.05 \pm 0.09$ & $5.28 \pm 0.22$ & $5.52 \pm 0.08$ & $5.07 \pm 0.12$ & $5.33 \pm 0.40$ \\
\hline & $6-8$ & $3.22 \pm 0.17$ & $3.07 \pm 0.12$ & $2.94 \pm 0.24$ & $3.20 \pm 0.18$ & $3.17 \pm 0.01$ \\
\hline & $8-10$ & $2.62 \pm 0.06$ & $2.60 \pm 0.10$ & $2.57 \pm 0.10$ & $2.61 \pm 0.16$ & $2.79 \pm 0.14$ \\
\hline & $10-12$ & $1.87 \pm 0.12$ & $1.85 \pm 0.02$ & $1.76 \pm 0.11$ & $1.76 \pm 0.07$ & $1.92 \pm 0.09$ \\
\hline & $12-14$ & $2.54 \pm 0.11^{\mathrm{b}}$ & $2.53 \pm 0.16^{\mathrm{b}}$ & $2.39 \pm 0.05^{\mathrm{b}}$ & $3.20 \pm 0.13^{\mathrm{a}}$ & $2.73 \pm 0.06^{\mathrm{b}}$ \\
\hline & $14-16$ & $5.28 \pm 0.21^{\mathrm{b}}$ & $7.12 \pm 0.57^{b}$ & $6.85 \pm 0.08^{\mathrm{b}}$ & $6.60 \pm 0.27^{b}$ & $14.46 \pm 3.33^{\mathrm{a}}$ \\
\hline & $16-18$ & $7.98 \pm 0.33$ & $7.79 \pm 1.10$ & $7.46 \pm 0.69$ & $8.29 \pm 1.14$ & $10.15 \pm 0.60$ \\
\hline & $18-20$ & $15.38 \pm 1.18^{\mathrm{a}}$ & $13.38 \pm 1.23^{\mathrm{ab}}$ & $14.96 \pm 2.23^{\mathrm{a}}$ & $13.52 \pm 1.97^{\mathrm{ab}}$ & $9.42 \pm 0.10^{\mathrm{b}}$ \\
\hline & Overall mean & $5.49 \pm 0.16^{\mathrm{b}}$ & $5.45 \pm 0.09^{\mathrm{b}}$ & $5.56 \pm 0.23^{b}$ & $5.53 \pm 0.20^{b}$ & $6.25 \pm 0.30^{\mathrm{a}}$ \\
\hline \multirow{5}{*}{$\begin{array}{c}\text { FCRe } \\
\text { (g feed/g egg) }\end{array}$} & $20-24$ & $5.92 \pm 0.31$ & $5.77 \pm 0.35$ & $5.64 \pm 0.22$ & $5.77 \pm 0.19$ & $5.78 \pm 0.29$ \\
\hline & $24-28$ & $4.51 \pm 0.10$ & $4.37 \pm 0.15$ & $4.23 \pm 0.19$ & $4.29 \pm 0.15$ & $4.34 \pm 0.07$ \\
\hline & $28-32$ & $3.90 \pm 0.10^{\mathrm{a}}$ & $3.82 \pm 0.19^{\mathrm{ab}}$ & $3.69 \pm 0.10^{\mathrm{ab}}$ & $3.27 \pm 0.08^{\mathrm{b}}$ & $3.94 \pm 0.04^{\mathrm{a}}$ \\
\hline & $32-36$ & $3.43 \pm 0.07$ & $3.37 \pm 0.10$ & $3.27 \pm 0.10$ & $3.21 \pm 0.06$ & $3.37 \pm 0.05$ \\
\hline & Overall mean & $4.44 \pm 0.04^{\mathrm{a}}$ & $4.33 \pm 0.07^{a b}$ & $4.21 \pm 0.02^{b}$ & $4.19 \pm 0.09^{b}$ & $4.36 \pm 0.08^{\mathrm{ab}}$ \\
\hline
\end{tabular}

a-----b Means \pm standard error in the same row with different superscripts are significantly different $(\mathrm{P} \leq 0.05)$.

Table 4. Means $\pm \mathrm{SE}$ of carcass characteristics as affected by flash light program

\begin{tabular}{llllll}
\hline \multicolumn{1}{c}{ Traits } & \multicolumn{5}{c}{ Treatments } \\
\cline { 2 - 6 } & \multicolumn{1}{c}{ C } & \multicolumn{1}{c}{ T1 } & \multicolumn{1}{c}{ T2 } & \multicolumn{1}{c}{ T3 } & \multicolumn{1}{c}{ T4 } \\
\hline Live body weight, $\mathbf{g}$ & $1348.5 \pm 30.2$ & $1360.7 \pm 26.5$ & $1354.5 \pm 36.5$ & $1344.1 \pm 28.9$ & $1339.1 \pm 26.8$ \\
Dressed carcass, \% & $66.9 \pm 0.61$ & $65.8 \pm 0.31$ & $67.0 \pm 0.52$ & $65.9 \pm 0.62$ & $66.4 \pm 0.72$ \\
Drumsticks, \% & $10.9 \pm 0.72$ & $10.8 \pm 0.28$ & $11.1 \pm 0.56$ & $10.4 \pm 0.92$ & $10.5 \pm 1.1$ \\
Femurs, \% & $11.4 \pm 0.39$ & $11.5 \pm 0.74$ & $11.6 \pm 0.34$ & $11.3 \pm 0.66$ & $11.3 \pm 0.72$ \\
Breast, \% & $14.0 \pm 0.73$ & $13.9 \pm 0.88$ & $14.2 \pm 0.66$ & $13.8 \pm 1.11$ & $13.6 \pm 0.92$ \\
Heart, \% & $0.82 \pm 0.02$ & $0.83 \pm 0.01$ & $0.84 \pm 0.03$ & $0.80 \pm 0.02$ & $0.81 \pm 0.01$ \\
Liver, \% & $3.43 \pm 0.08^{\mathrm{a}}$ & $3.42 \pm 0.10^{\mathrm{a}}$ & $3.44 \pm 0.06^{\mathrm{a}}$ & $2.84 \pm 0.11^{\mathrm{b}}$ & $2.86 \pm 0.06^{\mathrm{b}}$ \\
Gizzard, \% & $3.55 \pm 0.24$ & $3.54 \pm 0.26$ & $3.58 \pm 0.27$ & $3.42 \pm 0.18$ & $3.41 \pm 0.19$ \\
Giblets, \% & $7.80 \pm 0.24^{\mathrm{a}}$ & $7.79 \pm 0.30^{\mathrm{a}}$ & $7.86 \pm 0.26^{\mathrm{a}}$ & $7.06 \pm 0.23^{\mathrm{b}}$ & $6.08 \pm 0.22^{\mathrm{b}}$ \\
Abdominal fat, \% & $1.90 \pm 0.16^{\mathrm{a}}$ & $1.72 \pm 0.12^{\mathrm{ab}}$ & $1.51 \pm 0.11^{\mathrm{b}}$ & $1.52 \pm 0.14^{\mathrm{b}}$ & $1.50 \pm 0.08^{\mathrm{b}}$ \\
\hline
\end{tabular}
a-----b Means \pm standard error in the same row with different superscripts are significantly different $(\mathrm{P} \leq 0.05)$.

\section{Blood Parameters:}

No significant differences were observed for all blood parameters of treated chickens and those of the control, except that of the total lipids, AST and H/L
Ratio (Table 5). Total lipids were significantly lower in birds reared under light flashes programs of $9 \mathrm{hrs}$ or $12 \mathrm{hrs}$ light flashes as compared to the other groups. In chickens exposed to light flashes, the 
plasma lipids levels decrease as the number of light flashes increase. However, AST levels and H/L Ratios were significantly higher in birds reared under $12 \mathrm{CL} /$ day and $9 \mathrm{FL}+3 \mathrm{CL} /$ day or $12 \mathrm{FL} /$ day compared to light flashes programs $(3 \mathrm{FL}+9 \mathrm{CL} /$ day or $6 \mathrm{FL}+6 \mathrm{CL} /$ day). This may be a result of physiological stress that occurred after these treatments of exposing to longer hrs of light flashes. It has been postulated that continuous lighting reduces the opportunity for rest and sleep, thereby increasing fear reaction and physiological stress (Rozenboim et al., 1999), while birds provided with restricted light have lower physiological stress, improved immune response, increased sleep, increased overall activity, and improvement in bone metabolism and leg health (Classen et al., 2004). During the dark hours of the day, melatonin is released, it influence the secretion of several lymphokines that are integral to normal immune function by acting through other endocrine hormones (thyroid hormones) and increase the production of antibodies (Apeldoorn et al., 1999, Kliger et al., 2000 and Abbas et al., 2007). Broilers on intermittent photoperiods exhibited less stress, as measured by plasma corticosterone than counterparts on continuous light (Olanrewaju et al., 2006).

These results are in good agreement with those findings obtained by Ibrahim (2005) and Soliman et al. (2006) who concluded that there were no significant differences in blood constituents ( total protein, albumen and globulin) among birds reared under different light regimes. Also, Zulkifli et al. (1998), Moore and Siopes (2000) and Abbas et al. (2008) found that broilers reared under continuous light had a higher heterophil:lymphocyte ratio and experienced greater fear response than birds reared under a 12L:12D photoperiod. In the same line, ElFiky et al. (2008) indicated that serum total lipids was significantly higher for continuous light regime than constant $(16.2 \%)$ and intermittent (14.5\%) light, although serum total protein and cholesterol levels were not different among the different light regimes, revealing no physiological stress. The16L:8D program tended to reduce fearfulness and thus psychological stress (Bayram and Özkan, 2010). On the other hand, the result in the current study disagrees with those of Campo and Da'vila (2002), who reported that heterophil-to-lymphocyte ratio was unaffected by a nearly continuous lighting schedule (23L:1D). Birds on $23 \mathrm{~h}$ of light had a similar $\mathrm{H} / \mathrm{L}$ ratios compared with birds on the increasing photoperiod (Wang, et al., 2008). Onbasilar et al. (2007) found that $\mathrm{H} / \mathrm{L}$, cholesterol and triglyceride levels did not differ significant among different lighting groups. Also, El-Neney (2003) showed that broiler chicks reared under intermittent lighting system had significantly higher plasma cholesterol than those reared under continuous lighting system.

Table 5. Means \pm SE of blood parameters as affected by flash light program

\begin{tabular}{llllll}
\multicolumn{1}{c}{ Traits } & \multicolumn{5}{c}{ Treatments } \\
\cline { 2 - 6 } & \multicolumn{1}{c}{$\mathbf{C}$} & \multicolumn{1}{c}{ T1 } & \multicolumn{1}{c}{ T2 } & \multicolumn{1}{c}{ T3 } \\
\hline Total proteins (g/dl) & $5.00 \pm 0.002$ & $4.80 \pm 0.003$ & $4.82 \pm 0.004$ & $4.82 \pm 0.003$ & $4.98 \pm 0.005$ \\
Albumin (g/dl) & $3.00 \pm 0.03$ & $2.92 \pm 0.05$ & $2.95 \pm 0.02$ & $2.94 \pm 0.07$ & $3.05 \pm 0.04$ \\
Globulin (g/dl) & $2.00 \pm 0.02$ & $1.87 \pm 0.03$ & $1.86 \pm 0.04$ & $1.88 \pm 0.09$ & $1.92 \pm 0.06$ \\
Albumin: globulin ratio & $1.50 \pm 0.08$ & $1.56 \pm 0.10$ & $1.59 \pm 0.11$ & $1.56 \pm 0.10$ & $1.59 \pm 0.06$ \\
Total lipids (g/dl) & $15.03 \pm 0.75^{\mathrm{a}}$ & $14.97 \pm 0.62^{\mathrm{a}}$ & $13.56 \pm 0.58^{\mathrm{ab}}$ & $12.10 \pm 0.44^{\mathrm{b}}$ & $12.00 \pm 0.72^{\mathrm{b}}$ \\
Cholesterol (mg/dl) & $94.8 \pm 5.61$ & $98.7 \pm 4.92$ & $90.5 \pm 4.81$ & $90.2 \pm 5.54$ & $92.0 \pm 6.92$ \\
AST U/I & $11.98 \pm 0.58^{\mathrm{a}}$ & $9.01 \pm 0.84^{\mathrm{b}}$ & $9.06 \pm 0.66^{\mathrm{b}}$ & $10.44 \pm 0.72^{\mathrm{ab}}$ & $11.96 \pm 0.77^{\mathrm{a}}$ \\
ALT U/I & $4.36 \pm 0.15$ & $4.30 \pm 0.12$ & $4.14 \pm 0.08$ & $4.20 \pm 0.12$ & $3.98 \pm 0.09$ \\
H / L Ratio & $0.44 \pm 0.01^{\mathrm{a}}$ & $0.36 \pm 0.02^{\mathrm{b}}$ & $0.35 \pm 0.01^{\mathrm{b}}$ & $0.43 \pm 0.02^{\mathrm{a}}$ & $0.45 \pm 0.01^{\mathrm{a}}$ \\
\hline
\end{tabular}

a-----b Means \pm standard error in the same row with different superscripts are significantly different $(\mathrm{P} \leq 0.05)$.

\section{Egg production (EP):}

The data presented in Table (6) revealed no significant differences $(\mathrm{P}>0.05)$ in average of egg weight $(\mathrm{EW})$, egg number $(\mathrm{EN})$ and hen day egg production (HDP) among birds in the experimental groups at all studied ages except at 28-32 weeks of age in the EN and HDP. Generally, the total EN and overall mean of HDP for birds reared under light flashes program of $9 \mathrm{FL}+3 \mathrm{CL} /$ day (T3) significantly $(\mathrm{P} \leq 0.05)$ exceeded those of the control $(12 \mathrm{CL}), \mathrm{T} 1$ (3 FL+9CL/day), T2 (6 CL+6FL/day) and T4 (12 FL/day). Light plays a pivotal role regarding sight, stimulation of internal organs and initiation of hormone release (Scheideler, 1990). It is of practical importance to know how many hours of light must be given to the birds before and after age at first egg in order to maximize egg production. In relation to animal welfare, a minimum dark period for birds kept in captivity of $6 \mathrm{~h}$ is recommended (Prescott et al., 2003). Light stimulation induces an increase in the activity of the anterior lobe of the pituitary gland, which controls the release of the $\mathrm{LH}$ and $\mathrm{FSH}$ hormones, which stimulates gonadal development, eventually resulting in onset of lay (Lewis, 2006, Ingram, et al., 2007). Light program and duration is an important environmental factor affecting laying hens' egg production and quality (Lewis and Gous, 2006ab). Currently, light restriction programs are utilized. Pullets reared under short day produced significantly more eggs compared to those reared under long days (Ingram, et al., 2007). The obtained 
results for egg production coincided with the observations by Wanga et al. (2002), Lewis, et al. (2004), Ciacciariello and Gous (2005), Lewis, (2006), Lewis and Gous (2006b) and Lewis et al. (2007) who reported that light regimen significantly affect total egg production. Also, Li et al., (2008) showed that egg production of intermittence lighting of 8L: 4D: 4L: 8D was improved 5.60\% comparing with the general consecutive lighting regime (16L: 8D). Working with commercial egg laying hens, Lewis et al. (1997) observed that length of photostimulation did affect age at first egg, egg weight, egg production, egg output, and body weight. Yuan et al. (1994) found that hens subjected to 6:18, 8:16, and 10:14L: D exhibited significantly higher hen day egg production than those subjected to 4:20LD. However, HDP was significantly higher for females given long photoperiod than for those provided with short photoperiod (Lewis et al., 2010). However, Wanga et al. (2002) and Lewis et al. (2007) found that photoperiod had no effect on egg production. Similar results were reported by Ingram et al. (2007) and Lewis et al. (2010) who found no significant effects on egg weight due to photoperiods. However, egg weight was significantly affected by lighting regimes (Ciacciariello and Gous, 2005, Backhouse et al., 2004, Lewis, 2006, Lewis and Gous, 2006a, b). Mean egg weight is usually positively correlated with age at sexual maturity when gonadal development is modified by lighting (Lewis et al., 1997). Birds reared on 6-h day lengths, which matured about $7 \mathrm{~d}$ later than birds reared on 9 or $12 \mathrm{~h}$, produced significantly smaller eggs. Fox and Morris, (1958) and Lanson (1961) noted no significant effects egg production between birds receiving different flashing light regimens.

Table 6. Means $\pm \mathrm{SE}$ of egg production traits as affected by flash light program

\begin{tabular}{|c|c|c|c|c|c|c|}
\hline \multirow{2}{*}{ Traits } & \multirow{2}{*}{ Periods } & \multicolumn{5}{|c|}{ Treatments } \\
\hline & & $\mathbf{C}$ & T1 & $\mathbf{T 2}$ & T3 & T4 \\
\hline \multirow{5}{*}{$\begin{array}{c}\text { Egg } \\
\text { number } \\
\left(\begin{array}{c}\text { egg/hen/28 } \\
\text { days })\end{array}\right.\end{array}$} & P1 (20-24 w) & $10.93 \pm 0.49$ & $11.27 \pm 0.52$ & $11.50 \pm 0.47$ & $11.17 \pm 0.41$ & $11.13 \pm 0.39$ \\
\hline & P2 (24-28 w) & $14.53 \pm 0.43$ & $14.83 \pm 0.52$ & $15.47 \pm 0.68$ & $15.23 \pm 0.39$ & $14.83 \pm 0.28$ \\
\hline & P3 (28-32 w) & $17.33 \pm 0.43^{\mathrm{b}}$ & $17.57 \pm 0.7^{\mathrm{ab}}$ & $18.13 \pm 0.35^{\mathrm{ab}}$ & $19.00 \pm 0.43^{\mathrm{a}}$ & $16.83 \pm 0.15^{b}$ \\
\hline & P4 (32-36 w) & $19.73 \pm 0.46$ & $20.00 \pm 0.95$ & $20.57 \pm 0.55$ & $20.53 \pm 0.35$ & $19.70 \pm 0.60$ \\
\hline & Total & $62.53 \pm 0.86^{b}$ & $63.67 \pm 1.07^{a b}$ & $65.67 \pm 0.26^{\mathrm{ab}}$ & $65.93 \pm 0.88^{a}$ & $62.50 \pm 0.90^{b}$ \\
\hline \multirow{5}{*}{$\begin{array}{c}\text { Egg weight } \\
\text { (g) }\end{array}$} & P1 (20-24 w) & $42.00 \pm 0.15$ & $41.20 \pm 0.49$ & $41.93 \pm 0.35$ & $41.57 \pm 0.52$ & $41.80 \pm 0.49$ \\
\hline & P2 (24-28 w) & $42.83 \pm 0.54$ & $42.81 \pm 0.62$ & $42.60 \pm 0.61$ & $42.53 \pm 0.59$ & $42.90 \pm 0.61$ \\
\hline & P3 (28-32 w) & $44.41 \pm 0.36$ & $44.43 \pm 0.44$ & $44.54 \pm 0.34$ & $44.00 \pm 0.26$ & $44.24 \pm 0.28$ \\
\hline & P4 (32-36 w) & $45.53 \pm 0.52$ & $45.31 \pm 0.52$ & $45.33 \pm 0.43$ & $45.47 \pm 0.41$ & $45.53 \pm 0.41$ \\
\hline & Overall mean & $43.69 \pm 0.36$ & $43.44 \pm 0.06$ & $43.60 \pm 0.38$ & $43.39 \pm 0.42$ & $43.62 \pm 0.07$ \\
\hline \multirow{5}{*}{ HDP (\%) } & P1 (20-24 w) & $39.05 \pm 1.75$ & $40.24 \pm 1.86$ & $41.07 \pm 1.68$ & $39.88 \pm 1.46$ & $39.76 \pm 1.40$ \\
\hline & P2 (24-28 w) & $51.90 \pm 1.53$ & $52.98 \pm 1.86$ & $55.24 \pm 2.42$ & $54.40 \pm 1.40$ & $52.98 \pm 1.02$ \\
\hline & P3 (28-32 w) & $61.90 \pm 1.54^{b}$ & $62.74 \pm 2.54^{\mathrm{ab}}$ & $64.76 \pm 1.24^{\mathrm{ab}}$ & $67.86 \pm 1.52^{\mathrm{a}}$ & $60.12 \pm 0.52^{b}$ \\
\hline & P4 (32-36 w) & $70.48 \pm 1.65$ & $71.43 \pm 3.38$ & $73.45 \pm 1.96$ & $73.33 \pm 1.26$ & $70.36 \pm 2.15$ \\
\hline & Overall mean & $55.83 \pm 0.77^{b}$ & $56.85 \pm 0.95^{\mathrm{ab}}$ & $58.63 \pm 0.23^{\mathrm{ab}}$ & $58.87 \pm 0.79^{a}$ & $55.80 \pm 0.80^{b}$ \\
\hline
\end{tabular}

a-----b Means \pm standard error in the same row with different superscripts are significantly different $(\mathrm{P} \leq 0.05)$.

\section{Egg quality:}

Data presented in Table (7), indicated no significant differences $(\mathrm{P}>0.05)$ in average egg weight (AEW), egg shape index (ESI), Haugh Units $(\mathrm{HU})$, shell strength, egg problems and egg components (Albumen\%, Yolk\%, Shell\%) among all groups. However, there were significant differences $(\mathrm{P} \leq 0.05)$ in egg yolk index (EYI) and shell thickness (ST) values among the birds in the different experimental groups. The averages EYI of C, T1, T2 and $\mathrm{T} 3$ groups were significantly $(\mathrm{P} \leq 0.05)$ higher than those of the T4 group. With regard to ST, the average ST of T1 and T2 groups were significantly $(\mathrm{P} \leq 0.05)$ higher than those of the $\mathrm{C}, \mathrm{T} 3$ and $\mathrm{T} 4$ groups. Light is an important environmental factor affecting egg quality of laying hens. There is growing interest in the use of intermittent lighting regimes during the second half of production in chickens. The obtained results are in agreement with observations of Backhouse et al. (2004) who found that shell weight decreased and shell thickness index decreased for every hour increase in photoperiod. Also, Li et al.
(2008) showed that eggshell thickness, eggshell strength and egg yolk color did not significantly differ between 16L:8D and other lighting schedules. Egg size, egg shell quality, and albumen quality, as assessed by Haugh units, were not affected by intermittent light (Leeson, et al., 1982). Backhouse et al. (2004) found that egg weight increases, and shell weight and thickness index decrease with the lengthening of photoperiods. Lewis et al. (2010) found that egg weight was negatively related to photoperiod; however yolk quality was not significantly affected by photoperiod. Photoperiod during the laying period has been reported to significantly affect shell strength (Hawes et al., 1991 and Lewis et al., 2007). In contrast, Li et al. (2008) showed that Haugh units of the eggs produced by the birds under 16L: 8D increased by 4.14, and albumen heights increased by $0.3 \mathrm{~mm}$. Also, Lewis, et al. (2004), Lewis and Gous, (2006a,b), Lewis et al. (2007), Lewis et al. (2010) reported significant lighting treatments effects in the proportion of eggs laid on the floor and the number of cracked and dirty 
eggs. Shorter photoperiods were associated with higher incidences of floor laying and cracked eggs.

\section{Reproductive performance:}

The data presented in Table (8), show significant differences $(\mathrm{P} \leq 0.05)$ for the age at sexual maturity (female, male and BW of male), fertility, genital organs (ovary, testes percentages and follicle number) and semen quality (semen volume and sperm-cell concentration) among the experimental groups. However, there were no significant differences $(\mathrm{P}>0.05)$ in oviduct length, oviduct percentage, semen color \& $\mathrm{pH}$, reaction time and hatchability percentage among the birds in the different experimental groups.

Table 7. Means \pm SE of egg quality parameters and egg components as affected by flash light program

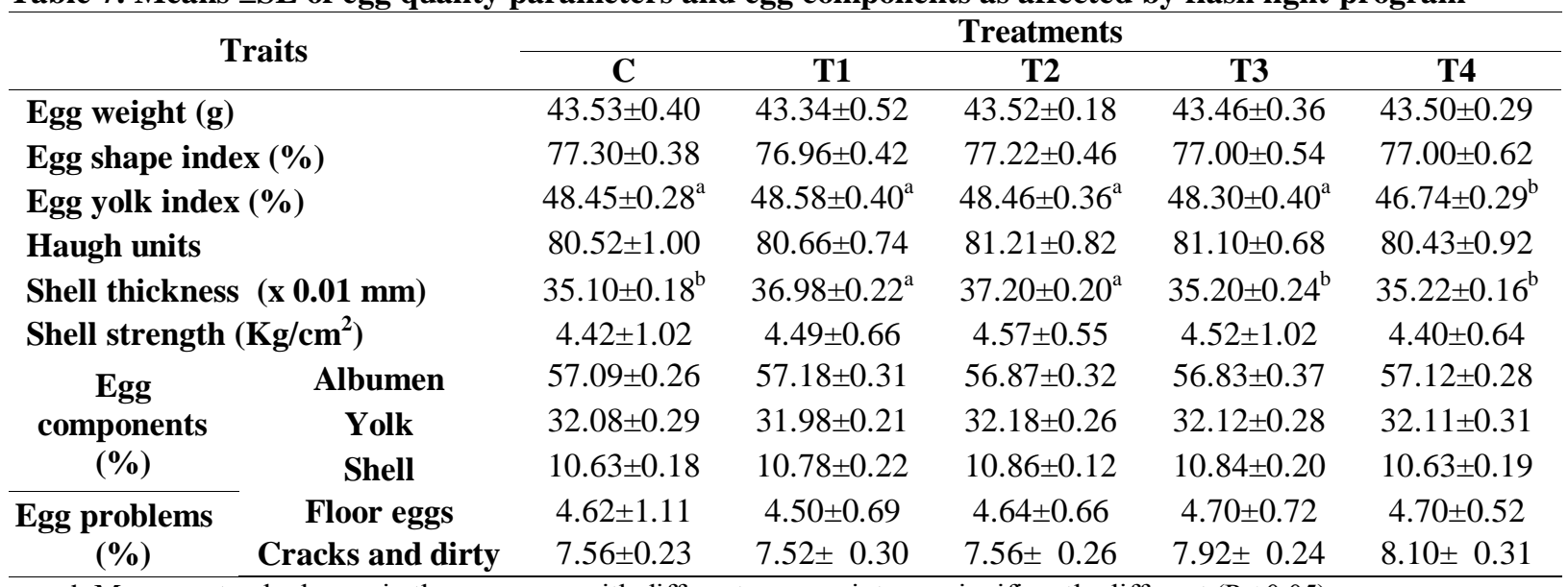

a-----b Means \pm standard error in the same row with different superscripts are significantly different $(\mathrm{P} \leq 0.05)$.

Physiologically, increasing day length stimulates hormone release in the brain which starts the process of maturation and reproduction performance (Olanrewaju et al., 2006).

Light stimulation induces an increase in the activity of the anterior lobe of the pituitary gland, which controls the release of the FSH. This stimulates the growth of ovarian follicles which develop to produce eggs (Ingram, et al., 2007). There is a relationship between melatonin (dark period) and gonadal size or sexual development (Singh and Haldar, 2007). Moreover, it has recently been postulated that melatonin, by stimulating expression of a newly discovered gonadotropin-inhibitory hormone, may provide an important photoperiodic signal to influence the reproductive axis of birds (Tsutsui et al., 2006). So, currently, light restriction programs are utilized. The present results are in agreement with the findings of Boon et al. (2000), Wanga et al. (2002), Lewis et al. (2004), Gous and Cherry (2004), Ciacciariello and Gous (2005), Lewis (2006), Lewis and Gous (2006b), Lewis et al. (2007) and Chen et al. (2007) who found that photoperiod significantly affect age at sexual maturity and egg number. However, photoperiod had no effect on BW at SM. Lewis and Gous (2006a) reported that body weight at first egg decreased by $20 \mathrm{~g}$ for each $1-\mathrm{d}$ advance in age at first egg for pullets of varying body weights. Also, Wanga et al. (2002) found that the fertility of geese eggs was improved for those receive in $14 \mathrm{~L}$ and $18 \mathrm{~L}$ groups. However, Shanawany (1993) noted that fertility and hatchability improved by long photoperiods in domestic fowl. While, Hawes et al. (1991) showed that intermittent light had no detrimental effects on fertility and hatchability. Also, fertility and hatchability were unaffected by either the adolescent or breeder light program (Ciacciariello and Gous 2005). On the other hand, it was stated that long photoperiods stimulate sexual maturation in birds (Lewis et al., 1997).

Excessively long and short photoperiods can restrict reproductive development in egg-type hens (Chen et al., 2007). Gous and Cherry (2004) found that broiler breeders require an increment in photoperiod to stimulate rapid gonadal development. Photostimulation is accompanied by increased $\mathrm{LH}$ and FSH secretion from the anterior pituitary gland which, in turn, initiates testicular development and Leydig cell proliferation (Henare, et al., 2011). In semen production, turkey males exposed to continuous or intermittent light regimes had minor differences in patterns of $\mathrm{LH}$ and testosterone secretion (Bacon et al., 2000). Chen et al. (2007) found that photoperiod had limited effect on ovarian follicle formation, whereas the photoperiod restricted ovary and oviduct full development. Ovary weights tended to be reduced, and lipid stores increased, relative to the longer photoperiod groups. Lewis et al. (2009b) found that testicular weight was significantly affected by photoperiod. However, Noirault et al. (2006) found that males in different photoperiod's groups had similar reproductive characteristics. They stated that plasma $\mathrm{LH}$ and testosterone concentrations were poor indicators of testis development and semen production, irrespective of age and photoperiod. Intermittent lighting support normal semen production in turkeys (Bacon et al., 1994). Also, Tyler et al. (2011) observed no photoperiodic effect 
on sperm concentration and production. Immature male turkeys exposed first to a short (6L:18D) and then to a long photoperiod (16L:8D) had marked increases in plasma concentrations of both $\mathrm{LH}$ and testosterone, this was followed by an increase in testis weight but not in semen output (Yang et al., 1998).

Table 8. Means $\pm \mathrm{SE}$ of reproductive performance as affected by flash light program

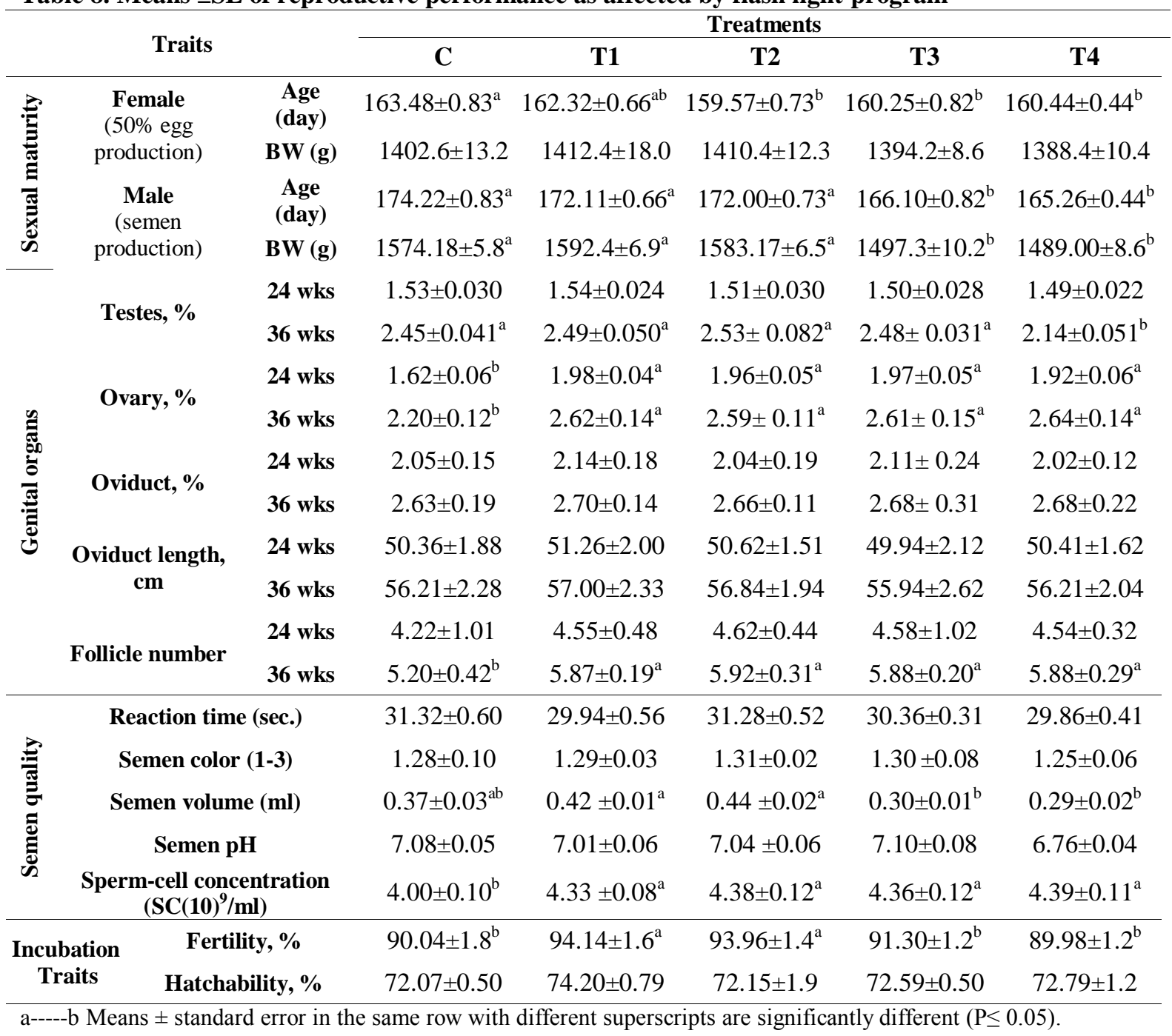

Livability, plumage conditions and bone measurements:

From the presented data in Table (9), it could be observed that there were no significant differences $(\mathrm{P}>0.05)$ in shank and keal bone length, leg problems, mortality rate and plumage conditions percentages among the birds in the different experimental groups for growing and laying periods. The photoperiodic regime can affect the physical activity, which affects energy expenditure and stimulate bone development, thereby improve the leg health of birds (Saiful et al., 2002, Olanrewaju et al., 2006). Sanotra et al. (2002) concluded that the lighting program not only reduced leg problems but also reduced chronic fear in the birds. The increased activity of the birds during the short periods of light may improve bone strength development (Buyse, et al., 1996). Similar findings were reported by Kristensen et al. (2006) who indicated that the light environment did not affect the severity of the gaitscore or hock-burns. On the contrary, the literature is well documented with evidence that lighting programs can reduce the incidence of leg disorders in broilers (Renden et al., 1996, Lewis et al., 2009a and Schwean-Lardner et al., 2012 and 2013). While, Tuleun et al. (2010) reported that continuous lighting significantly increased incidence of leg abnormalities and chronic fear in the birds. Ingram and Hatten (2000) and Hester et al. (2011) found that shank length was significantly decreased by light restriction. However, keel length was not significantly affected.

The absence of photoperiodic effects on mortality rate is in agreement with earlier findings for broiler breeders (Ciacciariello and Gous, 2005, Lewis et al., 2006, Lewis and Gous, 2006a, Lewis et al., 2007). Also, no significant differences between light treatments in mortality was reported (Rahimi et al., 
2005). The intermittent light program improved the immune performance by enhancing both humoral and cell-mediated response, which was a key factor in reducing mortality rate. Chickens reared under longer periods of darkness are reported to experience better health than their counterparts under long daylight conditions. In the literature, intermittent lighting programs have shown to increase livability and decrease metabolic diseases such as ascites, which is associated with pulmonary hypertension syndrome, sudden death syndrome, tibial dyschondroplasia and other skeletal disorders and improved immune system (Onbasilar et al., 2007). On the contrary, lower mortality has also been recorded in broilers reared under intermittent light compared to birds receiving long photoperiod (Rahimi, et al., 2005, Abbas et al., 2008, Lewis et al., 2009b and Gharib et al., 2008). Similar results were also reported by Shariatmadari and Moghadamian (2007).

Table 9. Means \pm SE of plumage conditions, bone measurements and mortality rate as affected by flash light

\begin{tabular}{lccccc}
\hline \multicolumn{1}{c}{ Traits } & \multicolumn{5}{c}{ Treatments } \\
\cline { 2 - 6 } & $\mathbf{C}$ & T1 & T2 & T3 & T4 \\
\hline Shank length $(\mathbf{c m})$ & $8.79 \pm 0.12$ & $8.83 \pm 0.13$ & $8.86 \pm 0.11$ & $8.82 \pm 0.10$ & $8.80 \pm 0.13$ \\
Keal bone length $(\mathbf{c m})$ & $6.90 \pm 0.24$ & $7.04 \pm 0.11$ & $6.98 \pm 0.20$ & $6.96 \pm 0.16$ & $7.00 \pm 0.30$ \\
Leg problems (\%) & $5.06 \pm 0.86$ & $4.80 \pm 1.04$ & $4.82 \pm 0.90$ & $3.92 \pm 0.46$ & $5.68 \pm 0.58$ \\
Plumage conditions & $2.70 \pm 0.16$ & $2.84 \pm 0.28$ & $2.75 \pm 0.28$ & $2.80 \pm 0.42$ & $2.69 \pm 0.19$ \\
Mortality rate (\%) & $10.88 \pm 1.13$ & $10.31 \pm 2.19$ & $12.45 \pm 1.30$ & $12.45 \pm 1.25$ & $13.08 \pm 1.18$ \\
\hline
\end{tabular}

No significant differences were observed $(\mathrm{P}>0.05)$.

Table 10. Economical efficiency for Dandarawi chickens as affected by flash light program

\begin{tabular}{|c|c|c|c|c|c|c|}
\hline & \multirow{2}{*}{ Items } & \multicolumn{5}{|c|}{ Treatments } \\
\hline & & $\mathbf{C}$ & T1 & $\mathbf{T} 2$ & $\mathbf{T 3}$ & T4 \\
\hline \multicolumn{7}{|c|}{ Total costs/ bird/L.E } \\
\hline \multirow{3}{*}{ Growing } & Electricity costs (L.E. /bird) & 0.62 & 0.45 & 0.32 & 0.24 & 0.12 \\
\hline & Feed costs (L.E) & 17.14 & 17.76 & 17.37 & 17.30 & 17.94 \\
\hline & Total costs/ bird/L.E & $\mathbf{1 7 . 7 6}$ & 18.21 & 17.69 & $\mathbf{1 7 . 5 4}$ & 18.06 \\
\hline \multirow{3}{*}{ Laying } & Electricity costs (L.E. /bird) & 0.80 & 0.60 & 0.45 & 0.35 & 0.25 \\
\hline & Feed costs (L.E) & 30.53 & 30.77 & 30.68 & 31.01 & 30.57 \\
\hline & Total costs/ bird/L.E & 30.88 & 31.37 & 31.13 & 31.81 & 30.82 \\
\hline \multicolumn{7}{|c|}{ Economical efficiency for growing } \\
\hline \multicolumn{2}{|c|}{ Selling price of bird at 20 weeks of age (L.E) } & 25.60 & 25.56 & 25.87 & 23.88 & 24.88 \\
\hline \multicolumn{2}{|c|}{ Net revenue/ bird/L.E (without *constant costs=25\%) } & 7.84 & 7.35 & 8.17 & 6.35 & 5.83 \\
\hline \multicolumn{2}{|c|}{ Economical efficiency/bird (EE) } & 0.46 & 0.41 & 0.47 & 0.37 & 0.32 \\
\hline \multicolumn{2}{|c|}{ Relative economical efficiency/bird (REE) } & 100.00 & 90.42 & 102.81 & 80.20 & 71.05 \\
\hline \multicolumn{7}{|c|}{ Economical efficiency for table eggs } \\
\hline \multirow{2}{*}{ Egg yield } & Egg mass/kg & 2.73 & 2.77 & 2.86 & 2.86 & 2.71 \\
\hline & Selling price as table egg/hen/L.E & 40.98 & 41.49 & 42.95 & 42.91 & 40.89 \\
\hline \multicolumn{2}{|c|}{ Net revenue/ bird/L.E (without *constant costs=25\%) } & 9.17 & 10.12 & 11.82 & 12.03 & 10.07 \\
\hline \multicolumn{2}{|c|}{ Economical efficiency/bird (EE) } & 0.30 & 0.33 & 0.39 & 0.39 & 0.33 \\
\hline \multicolumn{2}{|c|}{ Relative economical efficiency/bird (REE) } & 100.0 & 111.16 & 130.22 & 133.26 & 111.37 \\
\hline \multicolumn{7}{|c|}{ Economical efficiency for fertile eggs } \\
\hline \multirow{2}{*}{ Egg yield } & Fertile egg number/hen & 56.30 & 59.94 & 61.70 & 60.19 & 56.24 \\
\hline & Selling price as fertile egg/hen/L.E & 45.04 & 47.95 & 49.36 & 48.20 & 44.99 \\
\hline \multicolumn{2}{|c|}{ Net revenue/ bird/L.E (without $*$ constant costs $=25 \%)$} & 13.24 & 16.58 & 18.23 & 17.28 & 14.17 \\
\hline \multicolumn{2}{|c|}{ Economical efficiency/bird (EE) } & 0.43 & 0.54 & 0.59 & 0.57 & 0.46 \\
\hline \multicolumn{2}{|c|}{ Relative economical efficiency/bird (REE) } & 100.0 & 126.25 & 139.24 & 132.61 & 108.58 \\
\hline
\end{tabular}

Cost of $1 \mathrm{~kg}$ of dressed carcass $=28.00 \mathrm{~L} . \mathrm{E} . \quad$ Price of $1 \mathrm{~kg}$ table egg $=15.0 \mathrm{~L} . \mathrm{E} \quad$ Price of one fertile egg $=0.80 \mathrm{~L} . \mathrm{E}$

Price of $1 \mathrm{~kg}$ of growing ration = 3.00L.E Price of $1 \mathrm{~kg}$ of laying ration $=2.68 \mathrm{~L} . \mathrm{E} \quad \mathrm{L} . \mathrm{E}=$ Egyptian pound.

*Constant costs include: housing, labour, heating, cooling and treatment regimens. 
Economical efficiency (EE):

Results presented in Table (10) show that the economical efficiency of the T2 group exceeded the control group (C) by 2.81 . However, the T1, T3 and T4 groups decreased by $9.58,19.80$ and $28.95 \%$ compared to control group during the growing period. The economical efficiency of the T1, T2, T3 and T4 groups exceeded the control group (C) by $11.16,30.22,33.26$ and 11.37 as well as by 26.25 , $39.24,32.61$ and $8.58 \%$ for table egg and fertile egg production, respectively. The results of this study indicated that the shorter of light flashes regimen enhances economical efficient, this could be attributed to the superiority in immune functions and production performance of chickens. Also, it lowers the cost of electricity and room temperature. However, continuous light regimen seems to be a stressful program that induces elevation of $\mathrm{H} / \mathrm{L}$ ratio. This interesting findings have economic impacts on poultry farming due to lower feed intake in the chicks under intermittent light as well as a considerable saving in lighting (electricity) expense (Wang et al., 2008). In contrast, Oyedeji and Atteh (2005) found no significant interactions between factors for cost to benefit ratio. Also, Tuleun et al., (2010) reported that continuous lighting may improve weight gain, feed: gain ratio, reduced feed cost per kilogram and reduce the severity of leg abnormalities.

\section{CONCLUSION}

From the obtained results in this experiment, it could be concluded that the light flashes program of 3FL:9CL (T1) and 6FL:6CL (T2) were more economically efficient than the other treated groups. This could be attributed to the superiority of T1 and $\mathrm{T} 2$ in body weight performance, egg production, egg quality and lower mortality rates, as well as having adequate fertility. Nevertheless, flashes of light program were associated with less fat deposition which matches with the consumer's desire and health. From the practical point of view, light flashes can be only used in windowless rooms, otherwise this program can be used during the night. The light flashes program of $3 \mathrm{FL}: 9 \mathrm{CL}$ and $6 \mathrm{FL}: 6 \mathrm{CL}$ for growing and $8 \mathrm{FL}: 8 \mathrm{CL}$ and 12FL:4CL for laying Dandarawi chickens is highly recommended.

\section{REFERENCES}

Abbas A. O., A.K. Alm El-Dein, A.A. Desoky and M. A.A. Galal, 2008. The effects of photoperiod programs on broiler chicken performance and immune response. International J. of Poultry Science. 7: 665-671.

Abbas A. O., E. Ahmed Gehad, L. Gilbert, Hendricks, H.B.A. Gharib and M. M Mashaly, 2007. The Effect of lighting program and melatonin on the alleviation of the negative impact of heat stress on the immune response in broiler chickens. International J. of Poultry Science, 9: 651-660.
Al-Homidan A. A. and A. M. Petchey, 2000. The effects of length and color of light regimes on performance and carcass characteristics of broiler chickens. Egyptian Poultry Science, 21: 549-566.

Amakiri AO, OJ Owen and ES Etokeren, 2011. Broiler chicken's growth rate in three different nocturnal lighting regimes. African J. of food, agriculture, nutrition and development, 11: 1-8.

Apeldoorn E. J., J. W. Schrama, M. M. Mashaly and H. K. Parmentier, 1999. Effect of melatonin and lighting schedule on energy metabolism in broiler chickens. Poultry Science, 78: 223-229.

Backhouse D., P.D. Lewis and R.M. Gous, 2004. Constant photoperiods and eggshell quality in broiler breeder pullets. British Poultry Science, 46: 211-213.

Bacon W. L., B. A. Kurginski-Noonan, and J. Yang, 2000. Effects of environmental lighting on early semen production and correlated hormonal responses in turkeys. Poultry Science, 79: 16691678.

Bacon W. L., D. W. Long, K. Kurima and D. P. Chapman, 1994. Coordinate pattern of secretion of luteinizing hormone and testosterone in mature male turkeys under continuous and intermittent photoschedules. Poultry Science, 73: 864-870.

Bayraktar H., and A. Altan, 2012. The effects of spot lighting on broiler performance and welfare. J. of Animal and Veterinary Advances, 11: 1139-1144.

Bayram A. and S. Özkan, 2010. Effects of a 16-hour light, 8-hour dark lighting schedule on behavioral traits and performance in male broiler chickens. J. Applied Poultry Res., 19: 263-273.

Bölükbasi S. C. and H. Emsen, 2006. The Effect of diet with low protein and intermittent lighting on ascites induced by cold temperatures and growth performance in broilers. International J. of Poultry Science, 5: 988-991.

Boon Polly, G. Henk Visser, Serge Daan, 2000. Effect of photoperiod on body weight gain, and daily energy intake and energy expenditure in Japanese quail (Coturnix c. Japonica). Physiology \& Behavior.

Buyse J., P.C.M. Simons, F.M.G. Boshouwers and E. Decuypere 1996. Effect of intermittent lighting, light intensity and source on the performance and welfare of broilers. World's Poultry Science, 52: 121-130.

Campo J. L., and S. G. Da'vila, 2002. Effect of photoperiod on heterophil to lymphocyte ratio and tonic immobility duration of chickens. Poultry Science, 81: 1637-1639.

Chen H., R. L. Huang, H. X. Zhang, K. Q. Di, D. Pan and Y. G. Hou 2007. Effects of photoperiod on ovarian morphology and carcass traits at sexual maturity in pullets. Poultry Science, 86: 917-20.

Ciacciariello M., and R. M. Gous. 2005. To what extent can the age at sexual maturity of broiler breeders be reduced? S. African J. Animal Science 35: 73-82. 
Classen H.L., 2004. Day length affects performance, health and condemnations in broiler chickens. Proc. of the Australian Poult. Sci. Society, University of Sydney, Sydney, NSW.

Classen H.L., C.B. Annett, K.V. Schwean-Lardner, R. Gonda and D. Derow, 2004. The effects of lighting programmes with twelve hours of darkness per day provided in one, six or twelve hour intervals on the productivity and health of broiler chickens. British Poultry Science, 45: S3132.

Downs K. M., R. J. Lien, J. B. Hess, S. F. Bilgili, and W. A. Dozier, 2006. The effects of photoperiod length, light intensity, and feed energy on growth responses and meat yield of broilers. Applied Poultry Res J., 15: 406-416.

Duncan D.B., 1955. Multiple range and multiple tests. Biometrics, 11: 1-42.

Duve L.R., S. Steenfeldta, K. Thodberga and B.L. Nielsenb, 2011. Splitting the scotoperiod: effects on feeding behaviour, intestinal fill and digestive transit time in broiler chickens. British Poultry Science, 52: 1-10.

El-Fiky A., M. Soltan, F.H. Abdou, S. El- Samra and B. El- Neney, 2008. Effect of light regime and feeding frequency on some productive, physiological traits and hormonal profiles in broiler chicks. Egypt. Poultry Science, 28: 711743.

El-Neney B.A. 2003. Effect of light regimes and feed frequencies on broiler performance under Egyptian conditions. Ph.D. Thesis, Faculty of Agric., Minufiya Univ., Egypt.

Fox s. and T. R. Morris, 1958. Flash lighting for egg production. Nature, 182: 1752-1753.

Gharib H.B.A., A.A. Desoky, M.A. El-Menawey, 2008. The role of photoperiod and melatonin on alleviation of the negative impact of heat stress on broilers. International J. of Poultry Science, 7: 749-756.

Gous,R.M. and P. Cherry, 2004. Effect of body weight and lighting regimen and growth Curve to 20 weeks on laying performance in broiler breeders. British Poultry Science, 45: 445-452.

Hawes R.O., N. Lakshmanan and L.J. Kling, 1991. Effect of ahemeral light: dark cycles on egg production in early photostimulated brown-egg pullets. Poultry Science, 70: 1481-1486.

Henare S.J., M. Kikuchi, R.T. Talbot and J.F. Cockrem, 2011. Changes in plasma gonadotrophins, testosterone, prolactin, thyroxine and triiodothyronine concentrations in male Japanese quail (Coturnix coturnix japonica) of a heavy body weight line during photo-induced testicular growth and regression. British Poultry Science, 52: 782-791.

Hester P. Y., D. A. Wilson , P. Settar, J. A. Arango and N. P. O'Sullivan, 2011. Effect of lighting programs during the pullet phase on skeletal integrity of egg-laying strains of chickens. Poultry Science, 90: 1645-1651.
Houser J and B. Huber-Eicher 2004. Do domestic hens discriminate between familiar and unfamiliar conspecifics in the absence of visual cues? Applied Animal Behavior Science, 85: 65-76.

Ibrahim Faten A. A. 2005. Effect of light regimes on some productive and reproductive characteristics in Pekin drakes. Egyptian Poultry Science, 25 (2): 483-495.

Ingram D. R. and L. F. Hatten, 2000. Effects of light restriction on broiler performance and specific body structure measurements. J. Appl. Poultry Res., 9: 501-504.

Ingram D.R., L.F. Hatten and K.D. Homan, 2007. Reproductive performance of broiler breeders maintained on a photo schedule of only morning and evening artificial light in open-type houses. International J. of Poultry Science, 6: 424-426.

Johnson R.W., 1997. Inhibition of growth by proinflammatory cytokines: an integrated view. J. Animal Science, 75: 1244-1255.

Kliger C.A., A.E. Gehad, R.M. Hulet, W.B. Roush, H.S. Lillehoj and M.M. Mashaly, 2000. Effect of photoperiod and melatonin on lymphocyte activities in male broiler chickens. Poultry Science, 79: 18-25.

Kristensen H. H., J. M. Aerts, T. Leroy, C. M. Wathes, and D. Berckmans. 2006. Modelling the dynamic activity of broiler chickens in response to step-wise changes in light intensity. Applied Animal Behavour Science. 101: 125-143.

Kuhn E.R, V.M. Darras, C. Gysemans, E. Decuypere, L.R. Berghman and J. Buyse, (1996). The use of intermittent lighting in broiler raising. 2. Effects on the somatotrophic and thyroid axes and on plasma testosterone levels. Poultry Science, 75: 595-600.

Lanson, 1961. Effect of continuous, intermittent and flashing light on egg production, feed consumption and body weight. Poultry Science, 40: 1751-1756.

Leeson S., J.P.Walker and J. D.Summers, 1982. Performance of laying hens subjected to intermittent lighting initiated at 24 weeks of age. Poultry Science, 61: 567-568.

Lewis P. D., 2006. A review of lighting for broiler breeders. British Poultry Science, 47: 393-404.

Lewis P. D., and R. M. Gous. 2006c. Various photoperiods and Biomittent ${ }^{\mathrm{TM}}$ lighting during rearing for broiler breeders subsequently transferred to open-sided housing at 20 weeks. British Poultry Science, 47: 24-29.

Lewis P. D., R. Danisman and R. M. Gous, 2010. Photoperiods for broiler breeder females during the laying period. Poultry Science, 89: 108-114.

Lewis P. D., R. M. Gous, W. K. Ghebremariam, and P. J. Sharp, 2007. Broiler breeders do not respond positively to photoperiodic increments given during the laying period. British Poultry Science, 48: 245-252.

Lewis P.D, R. Danisman and R.M. Gous, 2009a. Photoperiodic responses of broilers. III. Tibial 
breaking strength and ash content. British Poultry Science, 50: 673-679.

Lewis P.D, R. Danisman and R.M. Gous, 2009 b. Photoperiodic responses of broilers. I. Growth, feeding behaviour, breast meat yield, and testicular growth. British Poultry Science, 50: 657-666.

Lewis, P. D. and R. M. Gous, 2006a. Constant and changing photoperiods in the laying period for broiler breeders allowed normal or accelerated growth during the rearing period. Poultry Science, 85: 321-325.

Lewis, P. D. and R. M. Gous, 2006b. Effect of final photoperiod and twenty-week body weight on sexual maturity and egg production in broiler breeders. Poultry Science, 85: 377-383.

Lewis, P. D., D. Backhouse, and R. M. Gous, 2004. Photoperiod and oviposition time in broiler breeders. British Poultry Science, 45: 561-564.

Lewis, P.D., G.C. Perry and T.R. Morris, 1997. Effect of size and timing of photoperiod increases on age at first egg and subsequent performance of two breeds of laying hens. Britiah Poultry Science, 38: 142-150.

Li Shen, Zhengxiang Shi, Baoming Li, Chaoyuan Wang, and He Ma, 2008. The effect of lighting programmes on egg production and quality of Beijing you-chicken. Qinghua East Road 17, Beijinng, 100083, China - Email:shizhx@cau.edu.cn.

Li, T. and H. Howland, 2003. The effects of constant and diurnal illumination of the pineal gland and the eyes on ocular growth in chicks. Iovs., 44: 3692-3697.

Lien R. J., L. B. Hooie and J. B. Hess, 2009. Influence of long-bright and increasing-dim photoperiods on live and processing performance of two broiler strains. Poultry Science, 88: 896903.

Mahmud, Saima A., Rafiullah and I. Ali, 2011. Effect of different light regimens on performance of broilers. The J. of Animal \& Plant Science, 21: 104-106.

Manser C.E., 1996. Effects of lighting on the welfare of domestic poultry: a review. Anim. Welfare 5: 341-360.

Moore, C. B., and T. D. Siopes, 2000. Effects of lighting conditions and melatonin supplementation on the cellular and humoral immune responses in Japanese quail Coturnix coturnix japonica.Gen. Comp. Endocrinol. 119: 95-104.

National Research Council (NRC), 1994. Nutrient Requirements of Poultry.9th rev. ed. National Academy Press, Washington, D.C.

Navara KJ and Nelson RJ. 2007. The dark side of light at night: Physiological, epidemiological and ecological consequences. J. of Pineal Research 43: 215-224

Noirault Je'ro^me, Jean-Pierre Brillard, Murray R. Bakst, 2006. Effect of various photoperiods on testicular weight, weekly sperm output and plasma levels of $\mathrm{LH}$ and testosterone over the reproductive season in male turkeys. Theriogenology, 66: 851-859.

Ohtani, S. and S. Lesson, 2000. The effect of intermittent lighting on metabolizable energy intake and heat production of male broilers. Poultry Science, 79: 167-171.

Olanrewaju H.A., J.P. Thaxton, W.A. Dozier, J. Purswell, W.B. Roush and S.L. Branton, 2006. A Review of lighting programs for broiler production. International J. of Poultry Science, 5: 301-308.

Onbaşılar E. E. , H. Erol1, Z. Cantekin and Ü. Kaya, 2007. Influence of intermittent lighting on broiler performance, incidence of tibial dyschondroplasia, tonic immobility, some blood parameters and antibody production. Asian-Aust. J. Animal Science, 20: 550-555

Oyedeji J.O. and J.O. Atteh, 2005. Effects of nutrient density and photoperiod on the performance and abdominal fat of broilers. International J. of Poultry Science, 4:149-152.

Pang, S. F., C. S. Pang, A. M. S. Poon, Q. Wan, Y. Song, and G. M. Brown, 1996. An overview of melatonin and melatonin receptors in birds. Poultry Avian Biology Rev., 7: 217-228.

Prescott, N. B., C. M. Wathes, and J. R. Jarvis. 2003. Light, vision, and the welfare of poultry. Animal Welfare 12: 269-288.

Rahimi, G., M. Rezaei, H. Hafezian and H. Saiyahzadeh, 2005. The effect of intermittent lighting schedule on broiler performance. International J. Poultry Science, 4: 396-398.

Renden, J.A., E.T. Moran, Jr. and S.A. Kincaid, 1996. Lighting programs for broilers that reduce leg problems without loss of performance or yield. Poultry Science, 75: 1345-1350.

Robinson, F. E., T. A. Wautier, R. T. Hardin, J. L. Wilson, M. Newcombe, and R. I. McKay, 1999. Effects of age at photostimulation on reproductive efficiency and carcass characteristics. 2. Egg-type hens. Canadian J. Animal Science, 76: 283-288.

Rozenboim, I., B. Robinzon and A. Rosenstrauch, 1999. Effect of light source and regimen on growing broilers. British Poultry Science, 40: 452-457.

Saiful I. M., Masanori Fujita and Toshio Ito, 2002. Effect of physical activity on heat production of white leghorn hen under different lighting regimes. J. Poultry Science, 39: 159-166.

Salisbury, G. W., VanDemark, N. K. and J. R., Lodge, 1985. Artificial insemination of cattle (2nd ed n.). CBS publishers and distributors, 485, Shahdara, Delhi.

Sanotra, C.S., Damkjer Lund, J. and K.S. Vestergaard, 2002 Influence of Light-Dark schedules and stocking density on behaviour, risk of leg problems and occurrence of chronic fear in broilers. British Poultry Science, 43: 344-354. 
SAS 1996. SAS User's Guide, statistics (6.2th ed.) Cary NC: SAS Institute Inc.

Scheideler, S. E. 1990. Research Note: Effect of various light sources on broiler performance and efficiency of production under commercial conditions. Poultry Science 69: 1030-1033.

Schwean-Lardner K., B. I. Fancher, H. L. Classen, 2012. Impact of daylength on the productivity of two commercial broiler strains. British Poultry Science, 53: 7-18, 2012.

Schwean-Lardner K., B. I. Fancher, S. Gomis, A. Van Kessel, S. Dalal and H. L. Classen, 2013. Effect of day length on cause of mortality, leg health, and ocular health in broilers. Poultry Science, 92:1-11.

Shanawany, M.M., 1993. Ahemeral lighting and reproductive efficiency in breeding flocks. World's Poultry Science, 49: 213-218.

Shariatmadari F. and A. A. Moghadamian, 2007. Effect of early feed restriction in combination with intermittent lighting during the natural scotoperiod on performance of broiler chicken. Isfahan University of Technology, 11: 363-374.

Shutze J. V., Jansen LS, Carver JS and WF Matson, 1996. Influence of various lighting regimes on the performance of growing chickens. Washington Agric. Expt. Sta. Tech. Bull., 36: www.reproductiononline.org.cgi/reprint/7/3/409.pdf. R.

Singh, S. S. and C. Haldar. 2007. Peripheral melatonin modulates seasonal immunity and reproduction of Indian tropical male bird Perdicula asiatica. Comp. Biochem. Physiol. A,Mol. Integr. Physiology, 146:446-450.

Soliman, M. M., B.A. El-Neney, M.A. Kalamah, and A.K. Alm El-Dein, 2006. Improving productive and physiological parameters of broilers using some light regimes and feed additives. Egypt. J. Animal Production, the 13th Conference of the Egypt. Society of Anim. Prod., Cairo, Egypt.1011 Dec, 269-286.

Tsutsui, K., T. Ubuka, H. Yin, T. Osugi, K. Ukena, G. E. Bentley, N. Ciccone, K. Inoue, V. S. Chowdhury, P. J. Sharp, and J. C. Wingfield. 2006. Mode of action and functional significance of avian gonadotropin-inhibitory hormone (GnIH): A review. J. Exp. Zoology, 305A:801806.
Tuleun C.D., P.C. Njoku and A.I. Okwori, 2010. Effect of dietary ascorbic acid on performance of broiler chickens exposed to different lighting regime. International J. of British Poultry Science, 9: 118-125.

Tyler Dr N.C., P.D. Lewisa and R.M. Gous, 2011. Reproductive status in broiler breeder males is minimally affected by a mid-cycle increase in photoperiod. British Poultry Science, 52: 140145.

Wang B., B. M. Rathgeber, T. Astatkie and J. L. MacIsaac, 2008. The stress and fear levels of microwave toe-treated broiler chickens grown with two photoperiod programs. Poultry Science, 87: $1248-1252$.

Wanga Sheng Der, Der-Fang Janb, Li-Tzu Yehc, Gwo-Chin Wua and Lih-Ren Chen, 2002. Effect of exposure to long photoperiod during the rearing period on the age at first egg and the subsequent reproductive performance in geese. Department of Animal Science, National ChungHsing University, Taichung, Taiwan.

Wineland, M.J. 2002. Fundamentals for managing light for poultry. In D.D. Bell and W.D. Weaver (Eds.). Commercial Chicken Meat and Egg Production (5th ed. pp.129-148). Norwell, MA: Kluwer Academic Publishers.

Yang, J., D. W. Long, N. Inpanbutr, and W. L. Bacon, 1998. Effects of photoperiod and age on secretory patterns of luteinizing hormone and testosterone and semen production in male domestic turkeys. Biology Reproduction 59: 1171-1179.

Yuan, T., R.J. Lien, and G.R. McDaniel, 1994. Effect of increasing rearing period body weight and early photostimulation on broiler breeder egg production. Poultry Science, 73: 792-800.

Zawilska JB, Lorenx A, Berezinska M, Vivien-Roels B, Pévet P and Skerne DJ. 2007. Photoperiod dependent changes in melatonin synthesis in the turkey pineal gland and retina. Poultry Science, 86: 1397-1405.

Zulkifli, I., A. Raseded, O.H. Syaadah and M.T.C. Morma, 1998. Daylength effects on stress and fear responses in broiler chickens. Asian-Aust. J. Animal Science, 11: 751-754. 


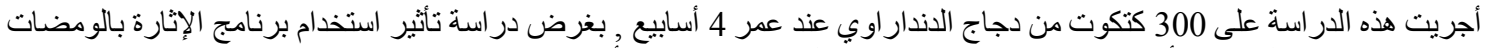

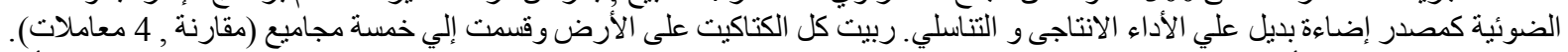

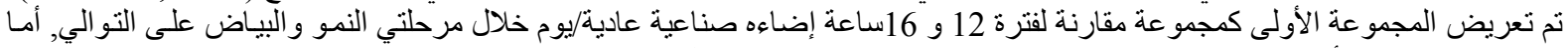

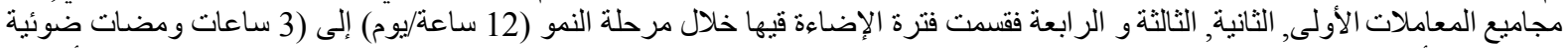

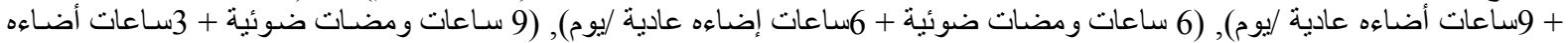

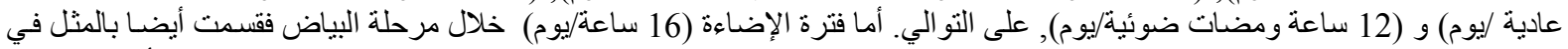

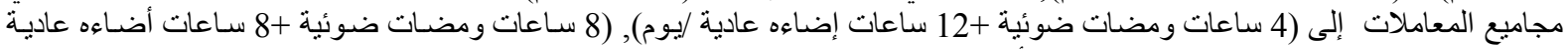

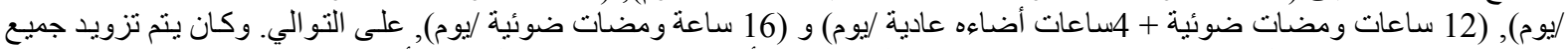

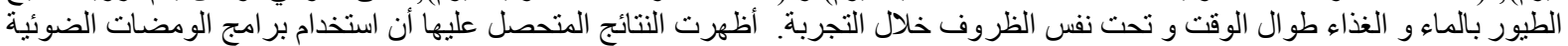

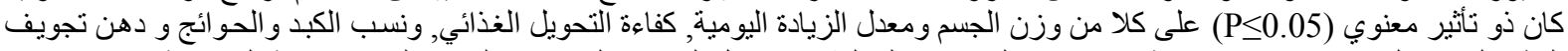

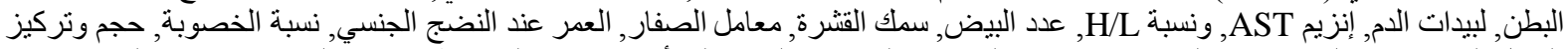

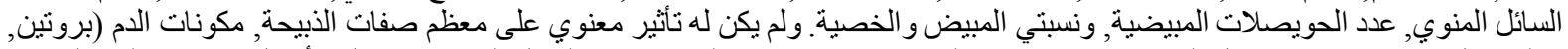

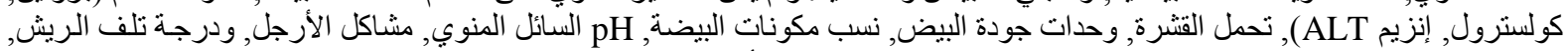

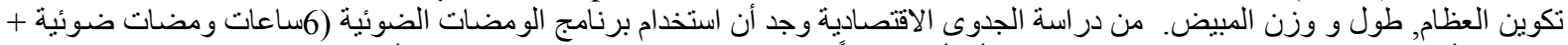

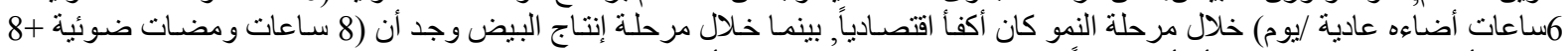

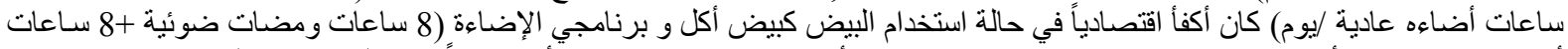

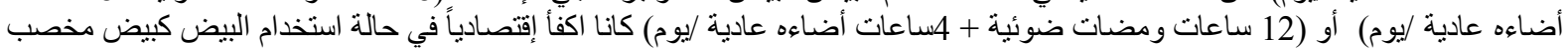

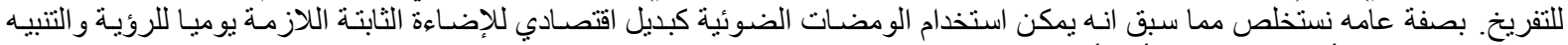

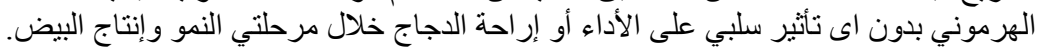

